\title{
New Exact Solutions for the (3+1)-Dimensional Generalized BKP Equation
}

\author{
Jun Su${ }^{1}$ and Genjiu $X u^{2}$ \\ ${ }^{1}$ School of Science, Xian University of Science and Technology, Xi'an 710054, China \\ ${ }^{2}$ Department of Applied Mathematics, Northwestern Polytechnical University, Xian 710072, China \\ Correspondence should be addressed to Jun Su; junsu99@126.com
}

Received 20 April 2016; Accepted 6 June 2016

Academic Editor: Nikos I. Karachalios

Copyright (C) 2016 J. Su and G. Xu. This is an open access article distributed under the Creative Commons Attribution License, which permits unrestricted use, distribution, and reproduction in any medium, provided the original work is properly cited.

\begin{abstract}
The Wronskian technique is used to investigate a $(3+1)$-dimensional generalized BKP equation. Based on Hirota's bilinear form, new exact solutions including rational solutions, soliton solutions, positon solutions, negaton solutions, and their interaction solutions are formally derived. Moreover we analyze the strangely mechanical behavior of the Wronskian determinant solutions. The study of these solutions will enrich the variety of the dynamics of the nonlinear evolution equations.
\end{abstract}

\section{Introduction}

In recent years, the problem of finding exact solutions of nonlinear evolution equations (NLEEs) is very popular for both mathematicians and physicists. Because seeking exact solutions of NLEEs is of great significance in nonlinear dynamics, many methods such as the inverse scattering transformation [1], Hirota's bilinear method [2], the Darboux transformation [3], the sine-cosine method [4], $G^{\prime} / G$-expansion method $[5,6]$, and the transformed rational function method [7] have been proposed. The Wronskian method which is based on the bilinear form of the NLEEs was proposed by Freeman and Nimmo in $[8,9]$. It is a fairly powerful tool to construct exact solutions of NLEEs in terms of the Wronskian determinant. By means of the method, the exact solutions of some NLEEs are obtained [10-16].

The study of the BKP equation has attracted a considerable size of research work. These equations were studied using the Hirota method, the multiple exp-function algorithm, the Pfaffian technique, Riemann theta functions, the extended homoclinic test approach, and Bäcklund transformation by many authors [17-26]. In this paper, based on the Wronskian method, the new exact solutions including rational solutions, soliton solutions, positon solutions, negaton solutions, and their interaction solutions of the (3+1)-dimensional generalized BKP equations are investigated.
In this paper, we will consider the following $(3+1)$ dimensional generalized BKP equation:

$$
u_{y t}-u_{x x x y}-3\left(u_{x} u_{y}\right)_{x}+6 u_{x x}-3 u_{z z}=0 .
$$

When $z=x$, this $(3+1)$-dimensional generalized BKP equation reduces to the BKP equation [27, 28]:

$$
u_{y t}-u_{x x x y}-3\left(u_{x} u_{y}\right)_{x}+3 u_{x x}=0 .
$$

By the dependent variable transformation

$$
u=2(\ln f)_{x}=\frac{2 f_{x}}{f}
$$

the (3+1)-dimensional generalized BKP equation (1) becomes a bilinear form

$$
\left(D_{y} D_{t}-D_{x}^{3} D_{y}+6 D_{x}^{2}-3 D_{z}^{2}\right) f \cdot f=0,
$$

where $D_{t}, D_{x}, D_{y}$, and $D_{z}$ are the Hirota operators [2]:

$$
\begin{gathered}
f\left(f_{y t}-f_{x x x y}+6 f_{x x}-3 f_{z z}\right)-f_{y} f_{t}+f_{x x x} f_{y} \\
+3 f_{x x y} f_{x}-3 f_{x x} f_{x y}-6 f_{x}^{2}+3 f_{z}^{2}=0 .
\end{gathered}
$$


We will show this $(3+1)$-dimensional generalized BKP equation has a class of Wronskian solutions with all generating functions for matrix entries satisfying a linear system of partial differential equations involving a free parameter. Rational solutions, solitons, positons, negatons, and interaction solutions to (1) among Wronskian determinant solutions are constructed and a few plots of particular solutions are made.

The paper is organized as follows. In Section 2, we derive a Wronskian formulation for the (3+1)-dimensional generalized BKP equation. In Section 3, Wronskian solutions to the $(3+1)$-dimensional generalized BKP equation are obtained. Section 4 presents the conclusion.

\section{A Wronskian Formulation}

The Wronskian technique is a powerful tool to construct exact solutions to bilinear differential or difference equations. To use the Wronskian technique, we adopt the compact notation introduced by Freeman and Nimmo $[8,9]$ :

$$
\begin{aligned}
W\left(\phi_{1}, \phi_{2}, \ldots, \phi_{N}\right) & =(\widehat{N-1} ; \Phi)=|\widehat{N-1}| \\
& =\left|\begin{array}{cccc}
\phi_{1}^{(0)} & \phi_{1}^{(1)} & \cdots & \phi_{1}^{(N-1)} \\
\phi_{2}^{(0)} & \phi_{2}^{(1)} & \cdots & \phi_{2}^{(N-1)} \\
\vdots & \vdots & \ddots & \vdots \\
\phi_{N}^{(0)} & \phi_{N}^{(1)} & \cdots & \phi_{N}^{(N-1)}
\end{array}\right|,
\end{aligned}
$$

where

$$
\begin{aligned}
\Phi & =\left(\phi_{1}, \ldots, \phi_{N}\right)^{T}, \\
\phi_{i}^{(0)} & =\phi_{i}, \\
\phi_{i}^{(j)} & =\frac{\partial^{j}}{\partial x^{j}} \phi_{i},
\end{aligned}
$$

$$
j \geq 1, \quad 1 \leq i \leq N .
$$

Solutions determined by $u=2(\ln f)_{x}$ with $f=|\widehat{N-1}|$ to the $(3+1)$-dimensional generalized BKP equation (1) are called Wronskian solutions.

Theorem 1. Assuming that a group of functions $\phi_{i}=$ $\phi_{i}(x, y, z, t), 1 \leq i \leq N$, satisfies the following linear conditions

$$
\begin{aligned}
-\phi_{i, x x} & =\sum_{j=1}^{N} \lambda_{i j}(t) \phi_{j}, \\
\phi_{i, y} & =k \phi_{i, x}, \\
\phi_{i, z} & =\sqrt{2} \phi_{i, x}, \\
\phi_{i, t} & =4 \phi_{i, x x x},
\end{aligned}
$$

where $k$ is an arbitrary nonzero constant, then the Wronskian determinant $f=|\widehat{N-1}|$ defined by (6) solves the bilinear equation (5).
Proof. Obviously, we have

$$
\begin{aligned}
f_{x}= & |\widehat{N-2}, N|, \\
f_{x x}= & |\widehat{N-3}, N-1, N|+|\widehat{N-2}, N+1|, \\
f_{x x x}= & |\widehat{N-4}, N-2, N-1, N| \\
& +2|\widehat{N-3}, N-1, N+1| \\
& +|\widehat{N-2}, N+2| .
\end{aligned}
$$

Using conditions (9), (10), and (11), we get that

$$
\begin{aligned}
f_{y} & =k|\widehat{N-2}, N|, \\
f_{x y} & =k(|\widehat{N-3}, N-1, N|+|\widehat{N-2}, N+1|), \\
f_{x x y} & =k(|\widehat{N-4}, N-2, N-1, N| \\
& +2|\widehat{N-3}, N-1, N+1|+|\widehat{N-2}, N+2|), \\
f_{x x x y} & =k(|\widehat{N-5}, N-3, N-2, N-1, N| \\
& +3|\widehat{N-4}, N-2, N-1, N+1| \\
& +2|\widehat{N-3}, N, N+1|+3|\widehat{N-3}, N-1, N+2| \\
& +|\widehat{N-2}, N+3|), \\
f_{z} & =\sqrt{2}|\widehat{N-2}, N|, \\
f_{z z} & =2(|\widehat{N-3}, N-1, N|+|\widehat{N-2}, N+1|), \\
f_{t} & =4(|\widehat{N-4}, N-2, N-1, N| \\
& -|\widehat{N-3}, N-1, N+1|+|\widehat{N-2}, N+2|), \\
f_{y t} & =4 k(|\widehat{N-5}, N-3, N-2, N-1, N| \\
& -|\widehat{N-3}, N, N+1|+|\widehat{N-2}, N+3|) .
\end{aligned}
$$

Under (8), it is not difficult to obtain [10]

$$
\begin{aligned}
& |\widehat{N-1}| \sum_{i=1}^{N} \lambda_{i i}(t)\left(\sum_{i=1}^{N} \lambda_{i i}(t)|\widehat{N-1}|\right) \\
& =\left(\sum_{i=1}^{N} \lambda_{i i}(t)|\widehat{N-1}|\right)^{2}=(|\widehat{N-2}, N+1| \\
& -|\widehat{N-3}, N-1, N|)^{2}=|\widehat{N-1}| \\
& \quad \cdot(|\widehat{N-5}, N-3, N-2, N-1, N| \\
& -|\widehat{N-4}, N-2, N-1, N+1| \\
& +2|\widehat{N-3}, N, N+1|-|\widehat{N-3}, N-1, N+2| \\
& +|\widehat{N-2}, N+3|) .
\end{aligned}
$$


Therefore,

$$
\begin{aligned}
f & \left(f_{y t}-f_{x x x y}+6 f_{x x}-3 f_{z z}\right)=3 k|\widehat{N-1}| \\
& \cdot(|\widehat{N-5}, N-3, N-2, N-1, N| \\
& -|\widehat{N-4}, N-2, N-1, N+1| \\
& -2|\widehat{N-3}, N, N+1|-|\widehat{N-3}, N-1, N+2| \\
& +|\widehat{N-2}, N+3|)=3 k(|\widehat{N-2}, N+1| \\
& \cdot|\widehat{N-3}, N-1, N|)^{2}-12 k|\widehat{N-3}, N, N+1| \\
& =12 k|\widehat{N-3}, N-1, N+1||\widehat{N-2}, N|-3 f_{x x} f_{x y} \\
& =-3 k(|\widehat{N-2}, N+1|-|\widehat{N-3}, N-1, N|)^{2} \\
& -12 k|\widehat{N-3}, N-1, N||\widehat{N-2}, N+1| .
\end{aligned}
$$

Substitution of the above results into (4) finally leads to the following Plücker relation:

$$
\begin{aligned}
\left(D_{y} D_{t}-D_{x}^{3} D_{y}+6 D_{x}^{2}-3 D_{z}^{2}\right) f \cdot f \\
=-12 k|\widehat{N-3}, N, N+1||\widehat{N-1}| \\
\quad+12 k|\widehat{N-3}, N-1, N+1||\widehat{N-2}, N| \\
\quad-12 k|\widehat{N-3}, N-1, N||\widehat{N-2}, N+1|=0 .
\end{aligned}
$$

Theorem 1 tells us that if a group of functions $\phi_{i}=$ $\phi_{i}(x, y, z, t), 1 \leq i \leq N$, satisfies the linear conditions in (8)(11), then we can get a solution $f=|\widehat{N-1}|$ to the bilinear $\mathrm{BKP}$ equation (4). The corresponding solution of (1) is

$$
u=2(\ln f)_{x}=\frac{2 f_{x}}{f}=\frac{2|\widehat{N-2}, N|}{|\widehat{N-1}|} .
$$

Remark 1. From the compatibility conditions $\phi_{i, x x t}=\phi_{i, t x x}$, $1 \leq i \leq N$, of conditions (8)-(11), we have the equality

$$
\sum_{j=1}^{N} \lambda_{i j}(t) \phi_{j}=0, \quad 1 \leq i \leq N
$$

and thus it is easy to see that the Wronskian determinant $W\left(\phi_{1}, \phi_{2}, \ldots, \phi_{N}\right)$ becomes zero if there is at least one entry $\lambda_{i j}$ satisfying $\lambda_{i j}(t) \neq 0$.

Remark 2. If the coefficient matrix $\Lambda=\left(\lambda_{i j}\right)$ is similar to another matrix $M=\left(\mu_{i j}\right)$ under an invertible constant matrix $P$, let us say $\Lambda=P^{-1} M P$, then $\widetilde{\Phi}=P \Phi$ solves

$$
\begin{aligned}
-\widetilde{\Phi}_{x x} & =M \widetilde{\Phi}, \\
\widetilde{\Phi}_{y} & =k \widetilde{\Phi}_{x},
\end{aligned}
$$

$$
\begin{aligned}
& \widetilde{\Phi}_{z}=\sqrt{2} \widetilde{\Phi}_{x}, \\
& \widetilde{\Phi}_{t}=4 \widetilde{\Phi}_{x x x},
\end{aligned}
$$

and the resulting Wronskian solutions to (1) are the same:

$$
\begin{aligned}
u(\Lambda) & =2 \partial_{x} \ln \left|\Phi^{(0)}, \Phi^{(1)}, \ldots, \Phi^{(N-1)}\right| \\
& =2 \partial_{x} \ln \left|P \Phi^{(0)}, P \Phi^{(1)}, \ldots, P \Phi^{(N-1)}\right|=u(M) .
\end{aligned}
$$

Based on Remark 1, we only need to consider case of (8)-(11) under $d \Lambda / d t=0$, that is, the following conditions:

$$
\begin{aligned}
-\phi_{i, x x} & =\sum_{j=1}^{N} \lambda_{i j}(t) \phi_{j}, \\
\phi_{i, y} & =k \phi_{i, x}, \\
\phi_{i, z} & =\sqrt{2} \phi_{i, x}, \\
\phi_{i, t} & =4 \phi_{i, x x x},
\end{aligned}
$$

where $\Lambda=\left(\lambda_{i j}\right)$ is an arbitrary real constant matrix. Moreover, Remark 2 tells us that an invertible constant linear transformation on $\Phi$ in the Wronskian determinant does not change the corresponding Wronskian solution, and thus, we only have to solve (21) under the Jordan form of $\Lambda$.

\section{Wronskian Solutions}

In principle, we can construct general Wronskian solutions of (1) associated with two types of Jordan blocks of the coefficient matrix $\Lambda$. But it is not easy. In this section we will present a few special Wronskian solutions to the generalized BKP equation, together with examples of exact solutions.

It is well known that the corresponding Jordan form of a real matrix

$$
\Lambda=\left[\begin{array}{cccc}
J\left(\lambda_{1}\right) & & & 0 \\
1 & J\left(\lambda_{2}\right) & & \\
& \ddots & \ddots & \\
0 & & 1 & J\left(\lambda_{m}\right)
\end{array}\right]_{n \times n}
$$

has the following two types of blocks:

(I)

$$
J\left(\lambda_{i}\right)=\left[\begin{array}{cccc}
\lambda_{i} & & & 0 \\
1 & \lambda_{i} & & \\
& \ddots & \ddots & \\
0 & & 1 & \lambda_{i}
\end{array}\right]_{k_{i} \times k_{i}}
$$

(II)

$$
\begin{aligned}
J\left(\lambda_{i}\right) & =\left[\begin{array}{cccc}
\Lambda_{i} & & & 0 \\
I_{2} & \Lambda_{i} & & \\
& \ddots & \ddots & \\
0 & & I_{2} & \Lambda_{i}
\end{array}\right]_{l_{i} \times l_{i}}, \\
\Lambda_{i} & =\left[\begin{array}{cc}
\alpha_{i} & -\beta_{i} \\
\beta_{i} & \alpha_{i}
\end{array}\right], I_{2}=\left[\begin{array}{ll}
1 & 0 \\
0 & 1
\end{array}\right],
\end{aligned}
$$


where $\lambda_{i}, \alpha_{i}, \beta_{i}$ are all real constants. The first type of blocks has the real eigenvalue $\lambda_{i}$ with algebraic multiplicity $k_{i}\left(\sum_{i=1}^{m} k_{i}=N\right)$, and the second type of blocks has the complex eigenvalue $\lambda_{i}^{ \pm}=\alpha_{i} \pm \beta_{i} \sqrt{-1}$ with algebraic multiplicity $l_{i}$.

3.1. Rational Solutions. Suppose $\Lambda$ has the first type of Jordan blocks. Without loss of generality, let

$$
J\left(\lambda_{1}\right)=\left[\begin{array}{cccc}
\lambda_{1} & & & 0 \\
1 & \lambda_{1} & & \\
& \ddots & \ddots & \\
0 & & 1 & \lambda_{1}
\end{array}\right]_{k_{1} \times k_{1}}
$$

In this case, if the eigenvalue $\lambda_{1}=0, J\left(\lambda_{1}\right)$ becomes of the following form:

$$
\left[\begin{array}{cccc}
0 & & & 0 \\
1 & 0 & & \\
& \ddots & \ddots & \\
0 & & 1 & 0
\end{array}\right]_{k_{1} \times k_{1}} .
$$

From condition (21), we get

$$
\begin{aligned}
& \phi_{1, x x}=0, \\
&-\phi_{i+1, x x}=\phi_{i}, \\
& \phi_{i, y}=k \phi_{i, x}, \\
& \phi_{i, z}=\sqrt{2} \phi_{i, x}, \\
& \phi_{i, t}=4 \phi_{i, x x x}, \\
& \quad i \geq 1 .
\end{aligned}
$$

Such functions $\phi_{i}(i \geq 1)$ are all polynomials in $x, y, z$, and $t$, and a general Wronskian solution to the (3+1)-dimensional generalized BKP equation (1)

$$
u=2 \partial_{x} \ln W\left(\phi_{1}, \phi_{2}, \ldots, \phi_{k_{1}}\right)
$$

is rational and is called a rational Wronskian solution of order $k_{1}-1$.

From (27), we solve $\phi_{1, x x}=0, \phi_{1, y}=k \phi_{1, x}, \phi_{1, z}=\sqrt{2} \phi_{1, x}$, $\phi_{1, t}=4 \phi_{1, x x x}$ and have

$$
\phi_{1}=c_{1}+c_{2}(x+k y+\sqrt{2} z),
$$

where $c_{1}, c_{2}$, and $k \neq 0$ are all real constants. Similarly, by solving $-\phi_{i+1, x x}=\phi_{1}, \phi_{i+1, y}=k \phi_{i+1, x}, \phi_{i+1, z}=\sqrt{2} \phi_{i+1, x}$, $\phi_{i+1, t}=4 \phi_{i+1, x x x}, i \geq 1$, then two special rational solutions of lower-order are obtained after setting some integral constants to be zero.

(1) Zero-Order. When $c_{1}=0, c_{2}=1, \phi_{1}=x+k y+\sqrt{2} z$, we have the corresponding Wronskian determinant $f=$ $W\left(\phi_{1}\right)=x+k y+\sqrt{2} z$ and the associated rational Wronskian solution of zero-order:

$$
u=2 \partial_{x} \ln W\left(\phi_{1}\right)=\frac{2}{x+k y+\sqrt{2} z} .
$$

(2) First-Order. Taking $c_{1}=0, c_{2}=-1, \phi_{1}=-(x+k y+\sqrt{2} z)$, we have $\phi_{2}=(1 / 6)(x+k y+\sqrt{2} z)^{3}+4 t$. In this case, the corresponding Wronskian determinant is $f=W\left(\phi_{1}, \phi_{2}\right)=$ $-(1 / 3)(x+k y+\sqrt{2} z)^{3}+4 t$, and the rational Wronskian solution of first-order reads

$$
u=2 \partial_{x} \ln W\left(\phi_{1}, \phi_{2}\right)=\frac{2(x+k y+\sqrt{2} z)^{2}}{(1 / 3)(x+k y+\sqrt{2} z)^{3}-4 t} .
$$

(3) Second-Order. Taking $\phi_{1}=x+k y+\sqrt{2} z, \phi_{2}=-(1 / 6)(x+$ $k y+\sqrt{2} z)^{3}-4 t$, we have $\phi_{3}=(1 / 120)(x+k y+\sqrt{2} z)^{5}+$ $2(x+k y+\sqrt{2} z)^{2} t$. Then the Wronskian determinant is $f=$ $W\left(\phi_{1}, \phi_{2}, \phi_{3}\right)=-(1 / 45)(x+k y+\sqrt{2} z)^{6}+(4 / 3)(x+k y+$ $\sqrt{2} z)^{3} t+16 t^{2}$, and the rational Wronskian solution of secondorder is given by

$$
\begin{aligned}
u & =2 \partial_{x} \ln W\left(\phi_{1}, \phi_{2}, \phi_{3}\right) \\
& =\frac{-(2 / 15)(x+k y+\sqrt{2} z)^{5}+4(x+k y+\sqrt{2} z)^{2} t}{-(1 / 45)(x+k y+\sqrt{2} z)^{6}+(4 / 3)(x+k y+\sqrt{2} z)^{3} t+16 t^{2}}
\end{aligned}
$$

3.2. Solitons, Positons, and Negatons. If the eigenvalue $\lambda_{i} \neq 0$, $J\left(\lambda_{i}\right)$ becomes of the following form:

$$
\left[\begin{array}{cccc}
\lambda_{i} & & & 0 \\
1 & \lambda_{i} & & \\
& \ddots & \ddots & \\
0 & & 1 & \lambda_{i}
\end{array}\right]_{k_{i} \times k_{i}}
$$

We start from the eigenfunction $\phi_{i}\left(\lambda_{i}\right)$ determined by

$$
\begin{aligned}
-\left(\phi_{i}\left(\lambda_{i}\right)\right)_{x x} & =\lambda_{i} \phi_{i}\left(\lambda_{i}\right), \\
\left(\phi_{i}\left(\lambda_{i}\right)\right)_{y} & =k\left(\phi_{i}\left(\lambda_{i}\right)\right)_{x}, \\
\left(\phi_{i}\left(\lambda_{i}\right)\right)_{z} & =\sqrt{2}\left(\phi_{i}\left(\lambda_{i}\right)\right)_{x}, \\
\left(\phi_{i}\left(\lambda_{i}\right)\right)_{t} & =4\left(\phi_{i}\left(\lambda_{i}\right)\right)_{x x x} .
\end{aligned}
$$

General solutions to this system in two cases of $\lambda_{i}>0$ and $\lambda_{i}<0$ read as

$$
\begin{aligned}
\phi_{i}\left(\lambda_{i}\right)= & C_{1 i} \sin \left(\sqrt{\lambda_{i}}\left(x+k y+\sqrt{2} z-4 t \lambda_{i}\right)\right) \\
& +C_{2 i} \cos \left(\sqrt{\lambda_{i}}\left(x+k y+\sqrt{2} z-4 t \lambda_{i}\right)\right), \\
\phi_{i}\left(\lambda_{i}\right)= & C_{3 i} \sinh \left(\sqrt{-\lambda_{i}}\left(x+k y+\sqrt{2} z-4 t \lambda_{i}\right)\right) \\
& +C_{4 i} \cosh \left(\sqrt{-\lambda_{i}}\left(x+k y+\sqrt{2} z-4 t \lambda_{i}\right)\right),
\end{aligned}
$$


respectively, where $C_{1 i}, C_{2 i}, C_{3 i}$, and $C_{4 i}$ are arbitrary real constants. By an inspection, we find that

$$
\begin{aligned}
& -\left[\begin{array}{c}
\phi_{i}\left(\lambda_{i}\right) \\
\frac{1}{1 !} \partial_{\lambda_{i}} \phi_{i}\left(\lambda_{i}\right) \\
\vdots \\
\frac{1}{\left(k_{i}-1\right) !} \partial_{\lambda_{i}}^{k_{i}-1} \phi_{i}\left(\lambda_{i}\right)
\end{array}\right]_{x x}
\end{aligned}
$$

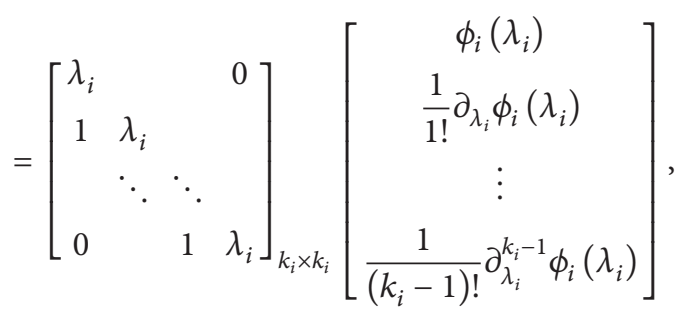

$$
\begin{aligned}
& \left(\frac{1}{j !} \partial_{\lambda_{i}}^{j} \phi_{i}\left(\lambda_{i}\right)\right)_{y}=k\left(\frac{1}{j !} \partial_{\lambda_{i}}^{j} \phi_{i}\left(\lambda_{i}\right)\right)_{x}, \\
& \left(\frac{1}{j !} \partial_{\lambda_{i}}^{j} \phi_{i}\left(\lambda_{i}\right)\right)_{z}=\sqrt{2}\left(\frac{1}{j !} \partial_{\lambda_{i}}^{j} \phi_{i}\left(\lambda_{i}\right)\right)_{x}, \\
& \left(\frac{1}{j !} \partial_{\lambda_{i}}^{j} \phi_{i}\left(\lambda_{i}\right)\right)_{t}=4\left(\frac{1}{j !} \partial_{\lambda_{i}}^{j} \phi_{i}\left(\lambda_{i}\right)\right)_{x x x}, \\
& 0 \leq j \leq k_{i}-1 .
\end{aligned}
$$

Therefore, through this set of eigenfunctions, we obtain a Wronskian solution to (1):

$$
\begin{aligned}
u= & 2 \partial_{x} \ln W\left(\phi_{i}\left(\lambda_{i}\right), \frac{1}{1 !} \partial_{\lambda_{i}} \phi_{i}\left(\lambda_{i}\right), \ldots, \frac{1}{\left(k_{i}-1\right) !}\right. \\
& \left.\cdot \partial_{\lambda_{i}}^{k_{i}-1} \phi_{i}\left(\lambda_{i}\right)\right)
\end{aligned}
$$

which corresponds to the first type of Jordan blocks with a nonzero real eigenvalue.

When $\lambda_{i}>0$, we get positon solutions [29], and when $\lambda_{i}<0$, we get negaton solutions [30]. If we suppose $\Lambda$ have $n$ different nonzero real eigenvalues, in which there are $l$ positive real eigenvalues and $n-l$ negative real eigenvalues, then a more general positon can be obtained by combining $l$ sets of eigenfunctions associated with different $\lambda_{i}>0$ :

$$
\begin{aligned}
u= & 2 \partial_{x} \ln W\left(\phi_{1}\left(\lambda_{1}\right), \frac{1}{1 !} \partial_{\lambda_{1}} \phi_{1}\left(\lambda_{1}\right), \ldots, \frac{1}{\left(k_{1}-1\right) !}\right. \\
& \cdot \partial_{\lambda_{1}}^{k_{1}-1} \phi_{1}\left(\lambda_{1}\right) ; \ldots ; \phi_{l}\left(\lambda_{l}\right), \frac{1}{1 !} \partial_{\lambda_{l}} \phi_{l}\left(\lambda_{l}\right), \ldots, \frac{1}{\left(k_{l}-1\right) !} \\
& \left.\cdot \partial_{\lambda_{l}}^{k_{l}-1} \phi_{l}\left(\lambda_{l}\right)\right) .
\end{aligned}
$$

Similarly, a more general negaton can be obtained by combining $n-l$ sets of eigenfunctions associated with different $\lambda_{i}<0$ :

$$
\begin{aligned}
u= & 2 \partial_{x} \ln W\left(\phi_{1}\left(\lambda_{1}\right), \frac{1}{1 !} \partial_{\lambda_{1}} \phi_{1}\left(\lambda_{1}\right), \ldots, \frac{1}{\left(k_{1}-1\right) !}\right. \\
& \cdot \partial_{\lambda_{1}}^{k_{1}-1} \phi_{1}\left(\lambda_{1}\right) ; \ldots ; \phi_{n-l}\left(\lambda_{n-l}\right), \frac{1}{1 !} \\
& \left.\cdot \partial_{\lambda_{n-l}} \phi_{n-l}\left(\lambda_{n-l}\right), \ldots, \frac{1}{\left(k_{n-l}-1\right) !} \partial_{\lambda_{n-l}}^{k_{n-l}-1} \phi_{n-l}\left(\lambda_{n-l}\right)\right) .
\end{aligned}
$$

This solution is called an $l$-positon of order $\left(k_{1}-1, k_{2}-\right.$ $\left.1, \ldots, k_{l}-1\right)$ or $n-l$-negaton of order $\left(k_{1}-1, k_{2}-1, \ldots, k_{n-l}-1\right)$. If $l=n$ or $l=0$, we simply say that it is an $n$-positon of order $n$ or an $n$-negaton of order $n$.

(1) Solitons. An $n$-soliton solution is a special $n$-negaton:

$$
u=2 \partial_{x} \ln W\left(\phi_{1}, \phi_{2}, \ldots, \phi_{n}\right),
$$

with $\phi_{i}$ being given by

$$
\begin{array}{ll}
\phi_{i}=\cosh \left(\sqrt{-\lambda_{i}}\left(x+k y+\sqrt{2} z-4 t \lambda_{i}\right)+\gamma_{i}\right), & \\
& i \text { odd, } \\
\phi_{i}=\sinh \left(\sqrt{-\lambda_{i}}\left(x+k y+\sqrt{2} z-4 t \lambda_{i}\right)+\gamma_{i}\right), & \\
& i \text { even, }
\end{array}
$$

where $\lambda_{1}<\lambda_{2}<\cdots<\lambda_{n}<0$ and $\gamma_{i}(1 \leq i \leq n)$ are arbitrary real constants. For example, a 1 -soliton to (1) is given by

$$
\begin{aligned}
u= & 2 \partial_{x} \ln W\left(\phi_{1}\right)=2 \partial_{x} \\
& \cdot \ln \left(\cosh \left(\sqrt{-\lambda_{1}}\left(x+k y+\sqrt{2} z-4 t \lambda_{1}\right)+\gamma_{1}\right)\right) \\
& =2 \sqrt{-\lambda_{1}} \tanh \left(\theta_{1}\right)
\end{aligned}
$$

where $\theta_{1}=\sqrt{-\lambda_{1}}\left(x+k y+\sqrt{2} z-4 t \lambda_{1}\right)+\gamma_{1}$.

Similarly, we have a 2 -soliton to (1):

$$
u=2 \partial_{x} \ln W\left(\cosh \left(\theta_{1}\right), \sinh \left(\theta_{2}\right)\right)=\frac{2\left(\lambda_{1}-\lambda_{2}\right)\left(\sinh \left(\theta_{1}-\theta_{2}\right)-\sinh \left(\theta_{1}+\theta_{2}\right)\right)}{\left(\sqrt{-\lambda_{1}}-\sqrt{-\lambda_{2}}\right) \cosh \left(\theta_{1}+\theta_{2}\right)-\left(\sqrt{-\lambda_{1}}+\sqrt{-\lambda_{2}}\right) \cosh \left(\theta_{1}-\theta_{2}\right)}
$$




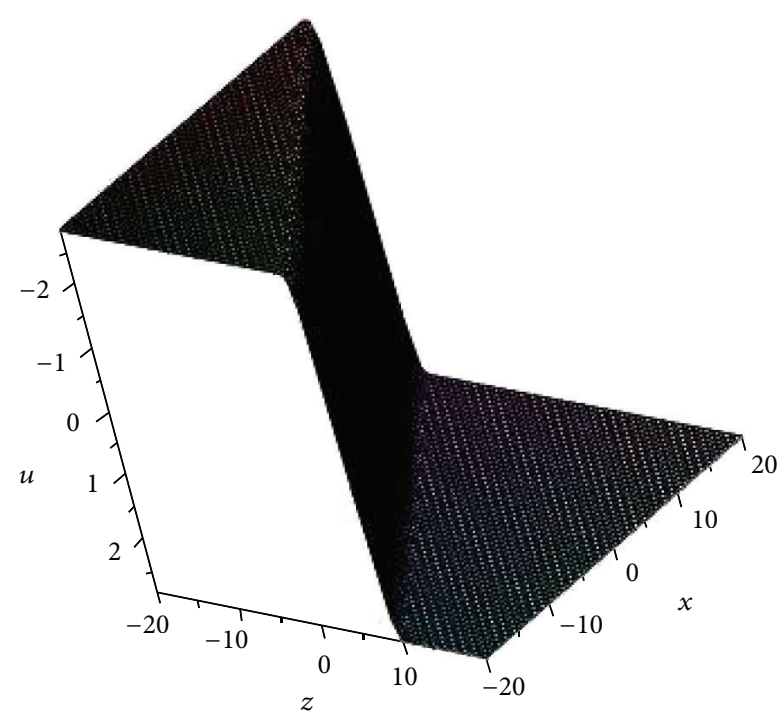

FIGURE 1: The shape of the 1-soliton to (1) with $\lambda_{1}=-2, y=0, t=1$, $\gamma_{1}=0$.

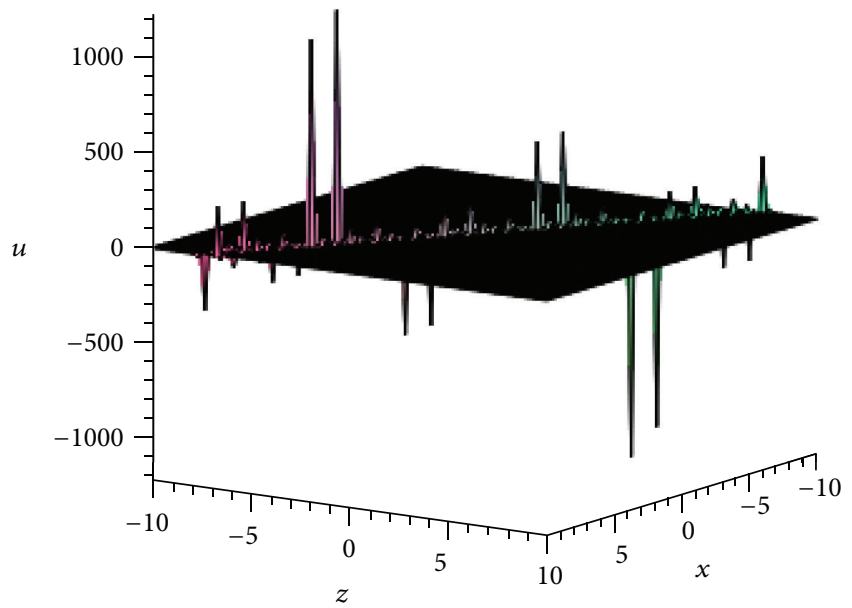

FIGURE 2: The shape of the 2-soliton to (1) with $\lambda_{1}=-4, \lambda_{2}=-1$, $y=0, t=0, \gamma_{1}=\gamma_{2}=0$.

where $\theta_{i}=\sqrt{-\lambda_{i}}\left(x+k y+\sqrt{2} z-4 t \lambda_{i}\right)+\gamma_{i}, i=1,2$. Figures 1 and 2 of three-dimensional plots show the $n$-soliton to (1) defined by (40) on the indicated specific regions, with specific values being chosen for the parameters.

(2) Positons. Two kinds of special positons of order $k_{1}-1$ are

$$
\begin{aligned}
& u=2 \partial_{x} \ln W\left(\phi, \partial_{\lambda} \phi, \ldots, \partial_{\lambda}^{k_{1}-1} \phi\right), \\
& \phi=\cos (\sqrt{\lambda}(x+k y+\sqrt{2} z-4 t \lambda)+\gamma(\sqrt{\lambda})), \\
& u=2 \partial_{x} \ln W\left(\phi, \partial_{\lambda} \phi, \ldots, \partial_{\lambda}^{k_{1}-1} \phi\right), \\
& \phi=\sin (\sqrt{\lambda}(x+k y+\sqrt{2} z-4 t \lambda)+\gamma(\sqrt{\lambda})),
\end{aligned}
$$

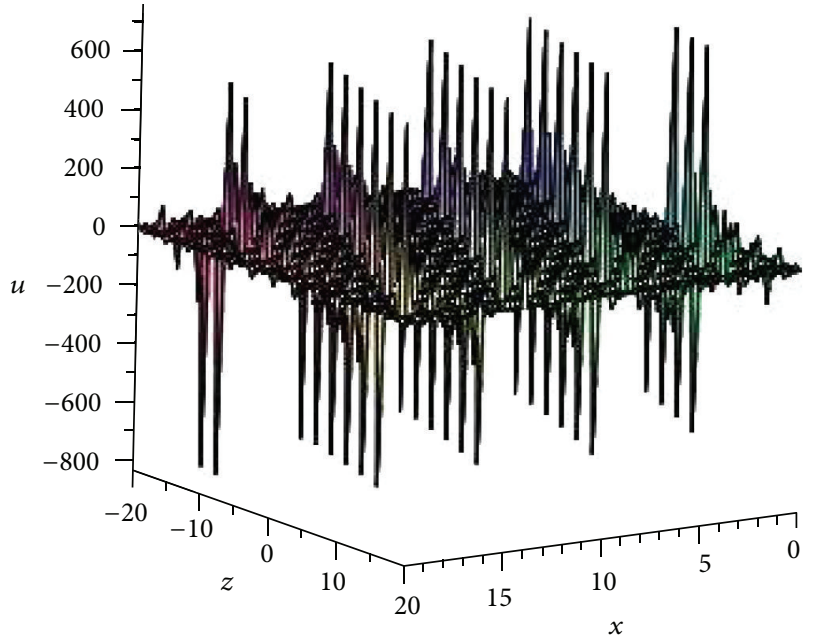

FIGURE 3: The shape of the 1-positon of zero-order to (1) with $\lambda_{1}=2$, $y=0, t=1, \gamma_{3}=0$.

where $\lambda>0$ and $\gamma$ is an arbitrary function of $\sqrt{\lambda}$. But these two kinds of positons are equivalent to each other, due to the existence of the arbitrary function $\gamma$.

When $\lambda_{1}>0$, a 1-positon of zero-order reads

$$
\begin{aligned}
u & =2 \partial_{x} \ln W\left(\phi_{1}\right) \\
& =2 \partial_{x} \ln \left(\cos \left(\sqrt{\lambda_{1}}\left(x+k y+\sqrt{2} z-4 t \lambda_{1}\right)+\gamma_{1}\right)\right) \\
& =-2 \sqrt{\lambda_{1}} \tan \left(\theta_{3}\right),
\end{aligned}
$$

where $\theta_{3}=\sqrt{\lambda_{1}}\left(x+k y+\sqrt{2} z-4 t \lambda_{1}\right)+\gamma_{3}$. And a 1-positon of first-order is

$$
\begin{aligned}
u & =2 \partial_{x} \ln W\left(\cos \left(\theta_{3}\right), \partial_{\lambda_{1}} \cos \left(\theta_{3}\right)\right) \\
& =\frac{4 \sqrt{\lambda_{1}}\left(1+\cos \left(2 \theta_{3}\right)\right)}{2 \sqrt{\lambda_{1}}\left(x+k y+\sqrt{2} z-12 t \lambda_{1}\right)+\sin \left(2 \theta_{3}\right)} .
\end{aligned}
$$

Figures 3 and 4 of three-dimensional plots show the special positons to (1) defined by (44) on the indicated specific regions, with specific values being chosen for the parameters.

(3) Negatons. Two kinds of special negatons of order $k_{1}-1$ are

$$
\begin{aligned}
& u=2 \partial_{x} \ln W\left(\phi, \partial_{\lambda} \phi, \ldots, \partial_{\lambda}^{k_{1}-1} \phi\right), \\
& \phi=\cosh (\sqrt{-\lambda}(x+k y+\sqrt{2} z-4 t \lambda)+\gamma(\sqrt{-\lambda})), \\
& u=2 \partial_{x} \ln W\left(\phi, \partial_{\lambda} \phi, \ldots, \partial_{\lambda}^{k_{1}-1} \phi\right), \\
& \phi=\sinh (\sqrt{-\lambda}(x+k y+\sqrt{2} z-4 t \lambda)+\gamma(\sqrt{-\lambda})),
\end{aligned}
$$

where $\lambda<0$ and $\gamma$ is an arbitrary function of $\sqrt{-\lambda}$. Similarly, these two kinds of negatons are equivalent to each other. 
When $\lambda_{1}<0$, a 1-negaton of first-order reads

$$
\begin{aligned}
u & =2 \partial_{x} \ln W\left(\cosh \left(\theta_{1}\right), \partial_{\lambda_{1}} \cosh \left(\theta_{1}\right)\right) \\
& =\frac{4 \sqrt{-\lambda_{1}}\left(1+\cosh \left(2 \theta_{1}\right)\right)}{2 \sqrt{-\lambda_{1}}\left(x+k y+\sqrt{2} z-4 t \lambda_{1}\right)+\sinh \left(2 \theta_{1}\right)},
\end{aligned}
$$

where $\theta_{1}=\sqrt{-\lambda_{1}}\left(x+k y+\sqrt{2} z-4 t \lambda_{1}\right)+\gamma_{1}$. And the 1-negaton of second-order is given by

$$
\begin{aligned}
u= & 2 \partial_{x} \ln W\left(\cosh \left(\theta_{1}\right), \partial_{\lambda_{1}} \cosh \left(\theta_{1}\right), \partial_{\lambda_{1}}^{2} \cosh \left(\theta_{1}\right)\right) \\
& =\frac{\left(-6 \lambda_{1} \delta_{1}+48 t \lambda_{1}^{2}\right) \sinh \left(\theta_{1}\right)-4 \lambda_{1} \sqrt{-\lambda_{1}} \delta_{1}^{2} \cosh \left(\theta_{1}\right)+6 \sqrt{-\lambda_{1}} \sinh ^{2}\left(\theta_{1}\right) \cosh \left(\theta_{1}\right)}{-2 \lambda_{1} \delta_{1}^{2} \sinh \left(\theta_{1}\right)+\left(24 t \lambda_{1} \sqrt{-\lambda_{1}}-\sqrt{-\lambda_{1}} \delta_{1}\right) \cosh \left(\theta_{1}\right)+\sinh \left(\theta_{1}\right) \cosh ^{2}\left(\theta_{1}\right)}
\end{aligned}
$$

where $\delta_{1}=x+k y+\sqrt{2} z-12 t \lambda_{1}$. Figures 5 and 6 of threedimensional plots show the special negatons to (1) defined by (48) on the indicated specific regions, with specific values being chosen for the parameters.

3.3. Interaction Solutions. We are now presenting examples of Wronskian interaction solutions among different kinds of Wronskian solutions to the $(3+1)$-dimensional generalized BKP equation (1).

Let us assume that there are two sets of eigenfunctions

$$
\begin{gathered}
\phi_{1}(\lambda), \phi_{2}(\lambda), \ldots, \phi_{l}(\lambda) \\
\psi_{1}(\mu), \psi_{2}(\mu), \ldots, \psi_{m}(\mu)
\end{gathered}
$$

associated with two different eigenvalues $\lambda$ and $\mu$, respectively. A Wronskian solution

$$
\begin{aligned}
u= & 2 \partial_{x} \ln W \\
& \cdot\left(\phi_{1}(\lambda), \phi_{2}(\lambda), \ldots, \phi_{l}(\lambda) ; \psi_{1}(\mu), \psi_{2}(\mu), \ldots, \psi_{m}(\mu)\right)
\end{aligned}
$$

is said to be a Wronskian interaction solution between two solutions determined by the two sets of eigenfunctions in (52).

In what follows, we would like to show a few special Wronskian interaction solutions. Let us first choose different sets of eigenfunctions:

$$
\begin{aligned}
& \phi_{\text {rational }}=x+k y+\sqrt{2} z \\
& \phi_{\text {soliton }}=\cosh \left(\sqrt{-\lambda_{1}}\left(x+k y+\sqrt{2} z-4 t \lambda_{1}\right)+\gamma_{1}\right), \\
& \phi_{\text {positon }}=\cos \left(\sqrt{\lambda_{2}}\left(x+k y+\sqrt{2} z-4 t \lambda_{2}\right)+\gamma_{2}\right),
\end{aligned}
$$

where $\lambda_{1}<0, \lambda_{2}>0$, and $\gamma_{i}(i=1,2)$ are arbitrary real constants.
Through three Wronskian interaction solutions between any two of a rational solution, a single soliton and a single positon read as

$$
\begin{aligned}
& u_{\mathrm{rs}}=2 \partial_{x} \ln W\left(\phi_{\text {rational }}, \phi_{\text {soliton }}\right) \\
& =\frac{-2 \lambda_{1}(x+k y+\sqrt{2} z) \cosh \left(\theta_{1}\right)}{\sqrt{-\lambda_{1}}(x+k y+\sqrt{2} z) \sinh \left(\theta_{1}\right)-\cosh \left(\theta_{1}\right)}, \\
& u_{\mathrm{rp}}=2 \partial_{x} \ln W\left(\phi_{\text {rational }}, \phi_{\text {positon }}\right) \\
& =\frac{2 \lambda_{2}(x+k y+\sqrt{2} z) \cos \left(\theta_{2}\right)}{\sqrt{\lambda_{2}}(x+k y+\sqrt{2} z) \sin \left(\theta_{2}\right)+\cos \left(\theta_{2}\right)}, \\
& u_{\mathrm{sp}}=2 \partial_{x} \ln W\left(\phi_{\text {soliton }}, \phi_{\text {positon }}\right) \\
& =\frac{2\left(\lambda_{2}-\lambda_{1}\right) \cosh \left(\theta_{1}\right) \cos \left(\theta_{2}\right)}{\sqrt{-\lambda_{1}} \sinh \left(\theta_{1}\right) \cos \left(\theta_{2}\right)+\sqrt{\lambda_{2}} \cosh \left(\theta_{1}\right) \sin \left(\theta_{2}\right)},
\end{aligned}
$$

where $\theta_{1}=\sqrt{-\lambda_{1}}\left(x+k y+\sqrt{2} z-4 t \lambda_{1}\right)+\gamma_{1}$ and $\theta_{2}=\sqrt{\lambda_{2}}(x+$ $\left.k y+\sqrt{2} z-4 t \lambda_{2}\right)+\gamma_{2}$.

One Wronskian interaction solution involving the three eigenfunctions is given by

$$
u_{\text {rsp }}=2 \partial_{x} \ln W\left(\phi_{\text {rational }}, \phi_{\text {soliton }}, \phi_{\text {positon }}\right)=\frac{2 q}{p},
$$

where

$$
\begin{aligned}
p= & (x+k y+\sqrt{2} z)\left(\lambda_{2} \sqrt{-\lambda_{1}} \sinh \left(\theta_{1}\right) \cos \left(\theta_{2}\right)\right. \\
& \left.+\lambda_{1} \sqrt{\lambda_{2}} \cosh \left(\theta_{1}\right) \sin \left(\theta_{2}\right)\right)+\left(\lambda_{1}-\lambda_{2}\right) \cosh \left(\theta_{1}\right) \\
& \cdot \cos \left(\theta_{2}\right), \\
q= & (x+k y+\sqrt{2} z) \sqrt{-\lambda_{1} \lambda_{2}}\left(\lambda_{1}-\lambda_{2}\right) \sinh \left(\theta_{1}\right) \\
& \cdot \sin \left(\theta_{2}\right)+\lambda_{1} \sqrt{-\lambda_{1}} \sinh \left(\theta_{1}\right) \cos \left(\theta_{2}\right)+\lambda_{2} \sqrt{\lambda_{2}} \\
& \cdot \cosh \left(\theta_{1}\right) \sin \left(\theta_{2}\right),
\end{aligned}
$$




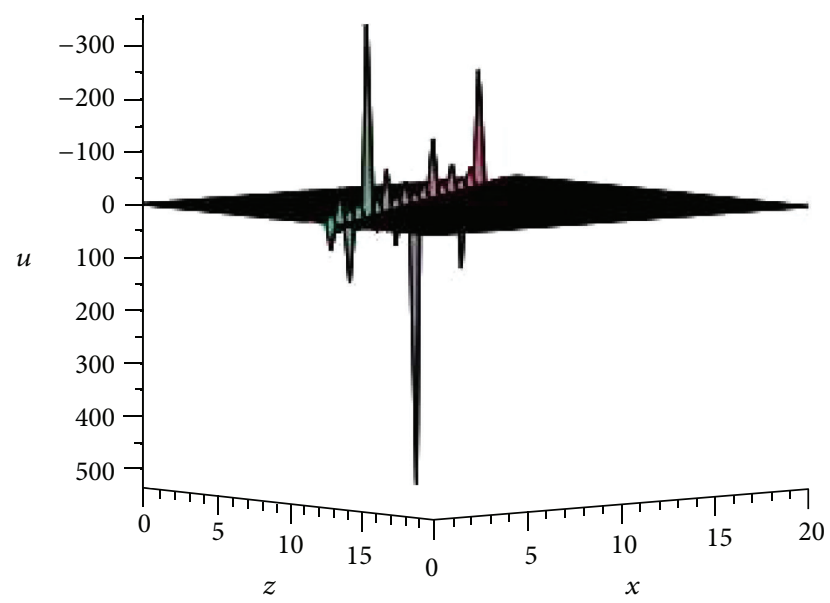

FIgURE 4: The shape of the 1-positon of first-order to (1) with $\lambda_{1}=$ $1.5, y=0, t=1, \gamma_{3}=0$.

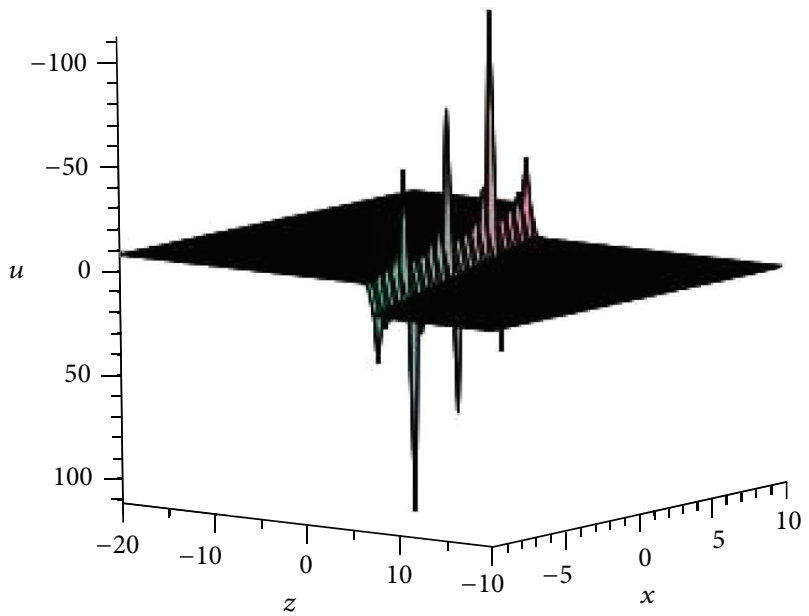

Figure 5: The shape of the 1-negaton of first-order to (1) with $\lambda_{1}=$ $-4, y=0, t=0, \gamma_{1}=0$.

$$
\begin{aligned}
& \theta_{1}=\sqrt{-\lambda_{1}}\left(x+k y+\sqrt{2} z-4 t \lambda_{1}\right)+\gamma_{1}, \\
& \theta_{2}=\sqrt{\lambda_{2}}\left(x+k y+\sqrt{2} z-4 t \lambda_{2}\right)+\gamma_{2} .
\end{aligned}
$$

Of course, we have more general Wronskian interaction solutions among three or more kinds of solutions such as rational solutions, positons, solitons, breathers, and negatons. Roughly speaking, it increases the complexities of rational solutions, positons, solitons, and negatons, respectively, to add zero, positive, negative eigenvalues to the spectrum of the coefficient matrix.

\section{Conclusion}

In summary we have extended the Wronskian method to a $(3+1)$-dimensional generalized BKP equation by its bilinear form. Moreover, we obtained some rational solutions, solitons, positons, negatons, and their interaction solutions to

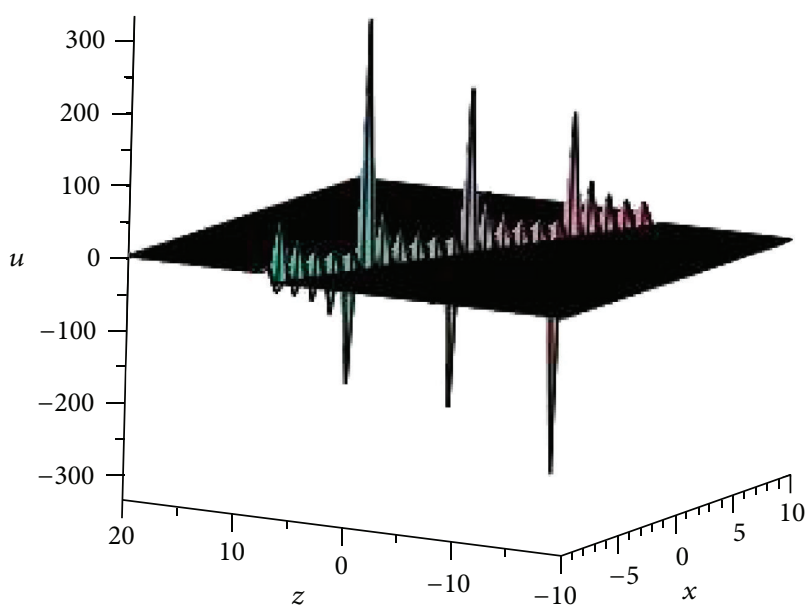

FIGURE 6: The shape of the 1-negaton of second-order to (1) with $\lambda_{1}=-1, y=0, t=0, \gamma_{1}=0$.

this equation by solving the systems of linear partial differential equations. All these show the richness of the solution space of the $(3+1)$-dimensional generalized BKP equation and the resulting solutions are expected to help understand wave dynamics in weakly nonlinear and dispersive media.

\section{Competing Interests}

The authors declare that they have no competing interests.

\section{Acknowledgments}

This work is supported by the National Natural Science Foundation of China (Grant nos. 11402194, 11501442, and 71271171), the Scientific Research Program Funded by Shaanxi Provincial Education Department (Program no. 2013JK0584), and the Science and Technology Research and Development Program in Shaanxi Province of China (Grant no. 2014KW03$01)$.

\section{References}

[1] M. J. Ablowitz and P. A. Clarkson, Nonlinear Evolution Equations and Inverse Scattering, Cambridge University Press, Cambridge, UK, 1991.

[2] R. Hirota, The Direct Methods in Soliton Theory, Cambridge University Press, Cambridge, UK, 2004.

[3] V. B. Matveev and M. A. Salle, Darboux Transformations and Solitons, Springer Series in Nonlinear Dynamics, Springer, Berline, Germany, 1991.

[4] A.-M. Wazwaz, "A sine-cosine method for handling nonlinear wave equations," Mathematical and Computer Modelling, vol. 40, no. 5-6, pp. 499-508, 2004.

[5] E. M. E. Zayed and K. A. Gepreel, “The $\left(G^{\prime} / G\right)$-expansion method for finding traveling wave solutions of nonlinear partial differential equations in mathematical physics," Journal of Mathematical Physics, vol. 50, no. 1, Article ID 013502, 12 pages, 2009. 
[6] A. R. Adem and C. M. Khalique, "New exact solutions and conservation laws of a coupled Kadomtsev-Petviashvili system," Computers \& Fluids, vol. 81, pp. 10-16, 2013.

[7] W.-X. Ma and J.-H. Lee, "A transformed rational function method and exact solutions to the $3+1$ dimensional JimboMiwa equation," Chaos, Solitons \& Fractals, vol. 42, no. 3, pp. 1356-1363, 2009.

[8] N. C. Freeman and J. J. Nimmo, "Soliton solutions of the Korteweg-de Vries and KADomtsev-Petviashvili equations: the Wronskian technique," Physics Letters. A, vol. 95, no. 1, pp. 1-3, 1983.

[9] J. J. C. Nimmo and N. C. Freeman, "A method of obtaining the $N$-soliton solution of the Boussinesq equation in terms of a Wronskian," Physics Letters A, vol. 95, no. 1, pp. 4-6, 1983.

[10] W.-X. Ma and Y. You, "Solving the Korteweg-de Vries equation by its bilinear form: Wronskian solutions," Transactions of the American Mathematical Society, vol. 357, no. 5, pp. 1753-1778, 2005.

[11] C.-X. Li, W.-X. Ma, X.-J. Liu, and Y.-B. Zeng, "Wronskian solutions of the Boussinesq equation-solitons, negatons, positons and complexitons," Inverse Problems, vol. 23, no. 1, pp. 279-296, 2007.

[12] X. Geng and Y. Ma, "N-soliton solution and its Wronskian form of a $(3+1)$-dimensional nonlinear evolution equation," Physics Letters A, vol. 369, no. 4, pp. 285-289, 2007.

[13] Z. Y. Yan, "Multiple solution profiles to the higher-dimensional Kadomtsev-Petviashvilli equations via Wronskian determinant," Chaos, Solitons and Fractals, vol. 33, no. 3, pp. 951-957, 2007.

[14] W.-X. Ma, C.-X. Li, and J. S. He, "A second Wronskian formulation of the Boussinesq equation," Nonlinear Analysis: Theory, Methods \& Applications, vol. 70, no. 12, pp. 4245-4258, 2009.

[15] W.-X. Ma, A. Abdeljabbar, and M. G. Asaad, "Wronskian and Grammian solutions to a $(3+1)$-dimensional generalized KP equation," Applied Mathematics and Computation, vol. 217, no. 24, pp. 10016-10023, 2011.

[16] Y. Tang, W.-X. Ma, W. Xu, and L. Gao, "Wronskian determinant solutions of the $(3+1)$-dimensional Jimbo-Miwa equation," Applied Mathematics and Computation, vol. 217, no. 21, pp. 8722-8730, 2011.

[17] W.-X. Ma and E. G. Fan, "Linear superposition principle applying to Hirota bilinear equations," Computers \& Mathematics with Applications, vol. 61, no. 4, pp. 950-959, 2011.

[18] A.-M. Wazwaz, "Two forms of (3+1)-dimensional B-type Kadomtsev-Petviashvili equation: multiple soliton solutions," Physica Scripta, vol. 86, no. 3, Article ID 035007, 2012.

[19] M. G. Asaad and W.-X. Ma, "Pfaffian solutions to a $(3+1)$ dimensional generalized B-type Kadomtsev-Petviashvili equation and its modified counterpart," Applied Mathematics and Computation, vol. 218, no. 9, pp. 5524-5542, 2012.

[20] W.-X. Ma and Z. N. Zhu, "Solving the $(3+1)$-dimensional generalized KP and BKP equations by the multiple exp-function algorithm," Applied Mathematics and Computation, vol. 218, no. 24, pp. 11871-11879, 2012.

[21] Y. L. Kang, Y. Zhang, and L. G. Jin, "Soliton solution to BKP equation in Wronskian form," Applied Mathematics and Computation, vol. 224, pp. 250-258, 2013.

[22] A.-M. Wazwaz, "Two B-type Kadomtsev-Petviashvili equations of $(2+1)$ and $(3+1)$ dimensions: multiple soliton solutions, rational solutions and periodic solutions," Computers \& Fluids, vol. 86, pp. 357-362, 2013.
[23] L. Cheng, Y. Zhang, and M.-J. Lin, "A Wronskian formulation of the $(3+1)$-dimensional generalized BKP equation," Physica Scripta, vol. 88, no. 1, Article ID 015002, 2013.

[24] A.-M. Wazwaz, "Variants of a $(3+1)$-dimensional generalized BKP equation: multiple-front waves solutions," Computers \& Fluids, vol. 97, pp. 164-167, 2014.

[25] Y. N. Tang and W. J. Zai, "New exact periodic solitary-wave solutions for the $(3+1)$-dimensional generalized KP and BKP equations," Computers \& Mathematics with Applications, vol. 70, pp. 2432-2441, 2015.

[26] S. Demiray and F. Taşcan, "Quasi-periodic solutions of $(3+1)$ generalized BKP equation by using Riemann theta functions," Applied Mathematics and Computation, vol. 273, pp. 131-141, 2016.

[27] E. Date, M. Jimbo, M. Kashiwara, and T. Miwa, "Transformation groups for soliton equations VI-KP hierarchies of orthogonal and symplectic type," Journal of the Physical Society of Japan, vol. 50, no. 11, pp. 3813-3818, 1981.

[28] E. Date, M. Jimbo, M. Kashiwara, and T. Miwa, "Transformation groups for soliton equations: IV. A new hierarchy of soliton equations of KP-type," Physica D: Nonlinear Phenomena, vol. 4, no. 3, pp. 343-365, 1982.

[29] V. B. Matveev, "Generalized Wronskian formula for solutions of the KdV equations: first applications," Physics Letters A, vol. 166, no. 3-4, pp. 205-208, 1992.

[30] C. Rasinariu, U. Sukhatme, and A. Khare, "Negaton and positon solutions of the KDV and mKDV Hierarchy," Journal of Physics A: Mathematical and General, vol. 29, no. 8, pp. 1803-1823, 1996. 


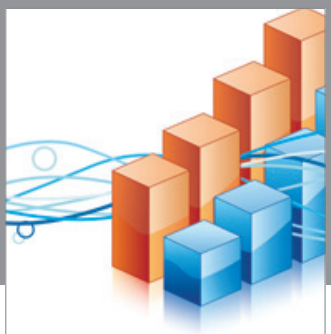

Advances in

Operations Research

vatem alat4

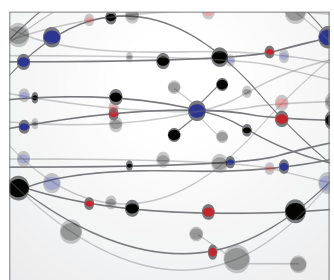

\section{The Scientific} World Journal
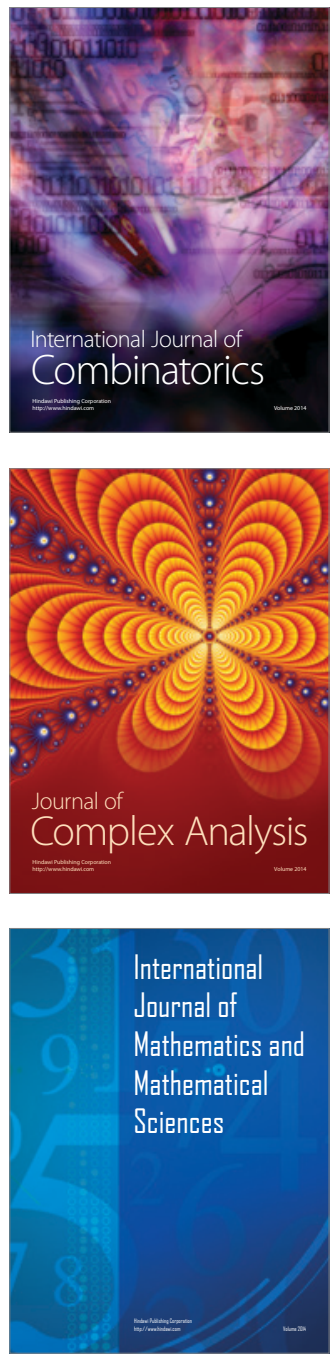
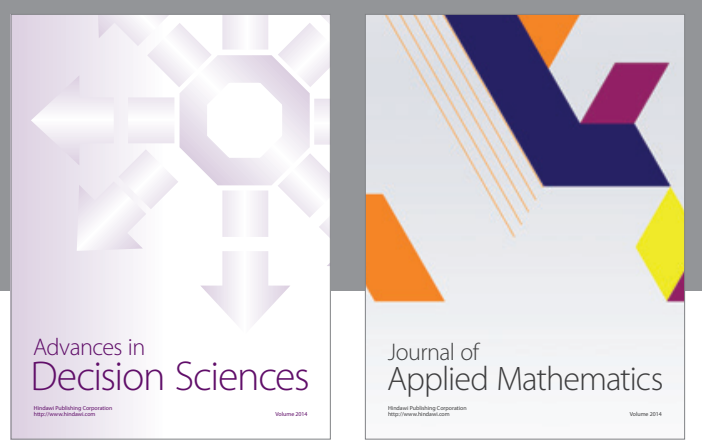

Algebra

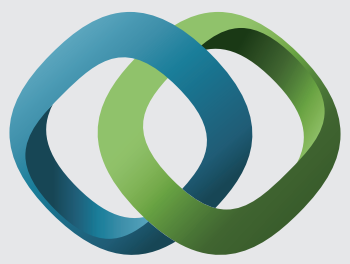

\section{Hindawi}

Submit your manuscripts at

http://www.hindawi.com
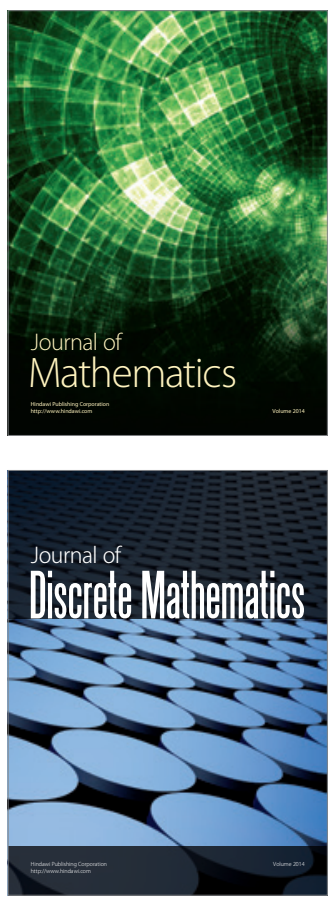

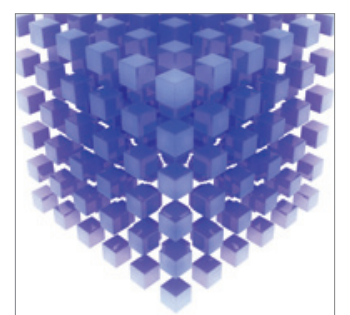

Mathematical Problems in Engineering
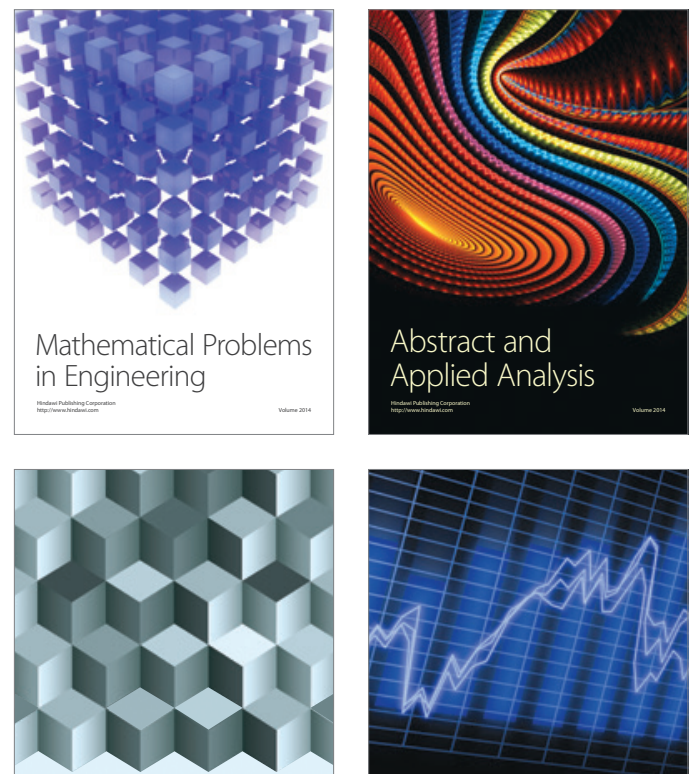

Journal of

Function Spaces

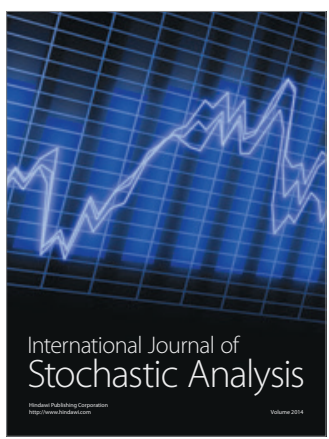

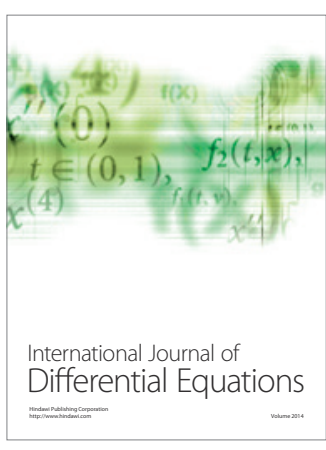
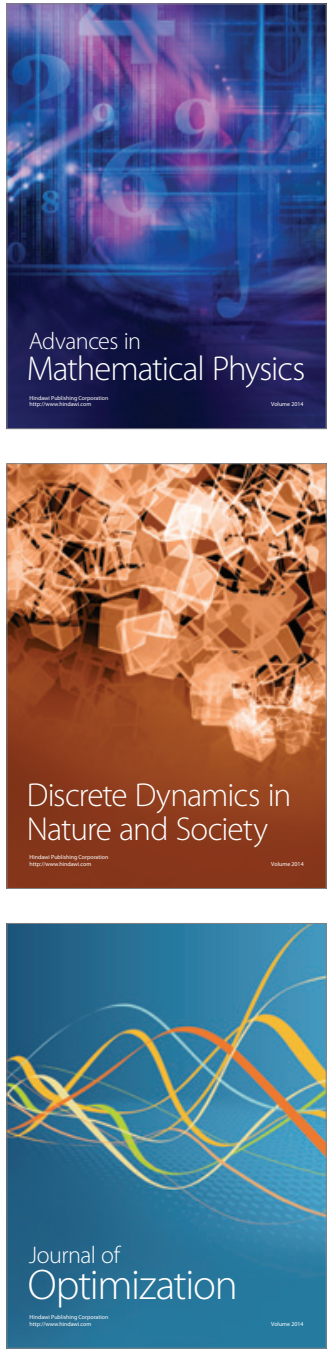